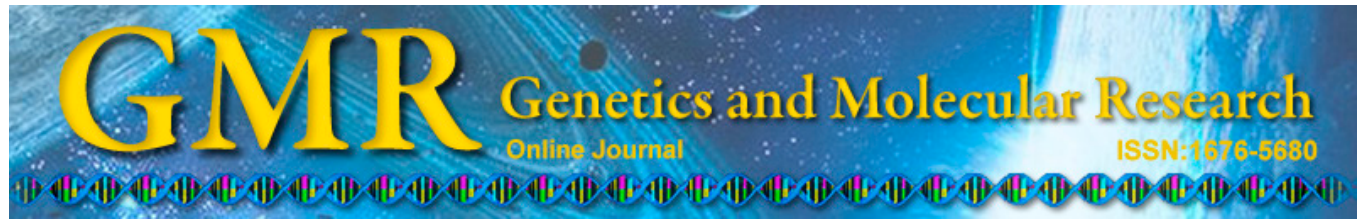

\title{
Single nucleotide polymorphisms in the mitochondrial displacement loop and age-at-onset of non-small cell lung cancer
}

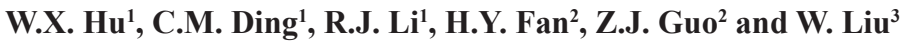 \\ ${ }^{1}$ Department of Respiratory Medicine, \\ The Fourth Hospital of Hebei Medical University, Shijiazhuang, China \\ ${ }^{2}$ Department of Gastroenterology and Hepatology, \\ The Fourth Hospital of Hebei Medical University, Shijiazhuang, China \\ ${ }^{3}$ Department of Oncology, The Fourth Hospital of Hebei Medical University, \\ Shijiazhuang, China \\ Corresponding author: W. Liu \\ E-mail: 13933852787@163.com
}

Genet. Mol. Res. 14 (1): 2512-2517 (2015)

Received January 14, 2014

Accepted June 3, 2014

Published March 30, 2015

DOI http://dx.doi.org/10.4238/2015.March.30.9

\begin{abstract}
Theassociations betweensinglenucleotidepolymorphisms (SNPs) in the displacement loop (D-loop) of mitochondrial DNA (mtDNA) and cancer risk and disease outcome have been extensively analyzed. We investigated the association between age-at-onset and SNPs in the mitochondrial D-loop using a population-based series of non-small cell lung cancer (NSCLC) patients. The D-loop region of mtDNA from NSCLC patients was amplified and sequenced. The ageat-onset curve of NSCLC patients was calculated using the KaplanMeier method at each SNP site and values were compared using the log-rank test. The SNP sites of nucleotides 200G/A and 16362T/C were identified to determine their association with age-at-onset of NSCLC using the log-rank test. The nucleotide $207 \mathrm{G} / \mathrm{A}$ was identified for its association with age-at-onset at a borderline significance level ( $\mathrm{P}$ $=0.060)$. We found that genetic polymorphisms in the D-loop were predictive markers for age-at-onset in NSCLC patients. Accordingly,
\end{abstract}


the analysis of genetic polymorphisms in the mitochondrial D-loop can be used to identify NSCLC patient subgroups at high risk of early onset.

Key words: Age-at-oneset; D-loop; Non-small cell lung carcinoma; Single nucleotide polymorphisms

\section{INTRODUCTION}

Lung cancer is the most frequent form of cancer and one of the leading causes of cancerrelated deaths worldwide, accounting for $30 \%$ of all cancer-related deaths. It is a major public health challenge, with an estimated 1.5 million patient deaths in 2010 (Parkin et al., 2001; Cao et al., 2011). The mortality rate of lung cancer in China is high, with approximately 400,000 patient deaths annually (Yang et al., 2009). Numerous risk factors for the development of lung cancer have been identified (Freedman et al., 2008; Lin et al., 2011; Toyooka et al., 2011), but few studies have focused on genetic factors associated with the age-at-onset of cancer.

The human mitochondrial genome is $16 \mathrm{~kb}$ in length and is a closed-circular duplex molecule containing 37 genes, including 2 ribosomal RNAs and a complete set of 22 tRNAs (Shadel and Clayton, 1997). Mitochondrial DNA (mtDNA) is thought to be more susceptible to DNA damage and acquires mutations at a higher rate than nuclear DNA because of the high levels of reactive oxygen species (ROS), the lack of protective histones, and the limited capacity for DNA repair in the mitochondria (Beal, 1996; DiMauro and Schon, 2001).

In cancer patients, sequence changes accumulate extensively in the mitochondrial displacement loop (D-loop) region, which is important for regulating both replication and expression of the mitochondrial genome. This region contains the leading-strand origin of replication and the main promoter for transcription (Taanman, 1999; Nashikawa et al., 2001; SanchezCespedes et al., 2001; Yoneyama et al., 2005). We sequenced the D-loop of 1122 base pairs in length (nucleotides 16,024-16,569 and 1-576), according to the mitochondria database (www. mitomap.org), in the blood from non-small cell lung cancer (NSCLC) patients, and identified cancer outcome associated with single-nucleotide polymorphisms (SNPs) using Kaplan-Meier methods (Ding et al., 2012a). In this study, we assessed the relationships between germline SNPs in the D-loop and age-at-onset in NSCLC patients.

\section{MATERIAL AND METHODS}

\section{Tissue specimens and DNA extraction}

Blood samples were collected from 106 NSCLC patients who received treatment at the Respiratory Department at the Fourth Hospital of Hebei University from 2001-2010. Genomic DNA was immediately extracted using the Wizard Genomic DNA extraction kit (Promega, Madison, WI, USA) and stored at $-20^{\circ} \mathrm{C}$. All procedures were approved by the Human Tissue Research Committee at the hospital. Informed consent was obtained from all participants before enrollment.

\section{Polymerase chain reaction (PCR) amplification and sequence analysis}

The forward primer 5'-CCCCATGCTTACAAGCAAGT-3' (nucleotides 16190- 
16209) and reverse primer 5'-GCTTTGAGGAGGTAAGCTAC-3' (nucleotides 602-583) were used to amplify a 982-base pair sequence from the mtDNA D-loop region. PCR was performed according to the protocol of PCR Master Mix Kit (Promega) and purified prior to sequencing. Cycle sequencing was performed using the Dye Terminator Cycle Sequencing Ready Reaction Kit (Applied Biosystems, Foster City, CA, USA). The products were separated on the ABIPRISM Genetic Analyzer 3100 (Applied Biosystems). Polymorphisms were confirmed by repeated analyses of both strands.

\section{Statistical analysis}

The age-at-onset curve of NSCLC patients was calculated using the Kaplan-Meier method at each SNP site and compared using the log-rank test. All statistical analyses were conducted using the SPSS 15.0 software package (SPSS Company; Chicago, IL, USA). P < 0.05 was considered to be statistically significant.

\section{RESULTS}

A total of 106 NSCLC patients, including 35 diagnosed with squamous cell carcinoma and 71 with adenocarcinoma, were enrolled in this study. The age-at-onset distribution of NSCLC patients is listed in Table 1. Patients analyzed included 7 patients aged less than 40 years, 11 patients aged 40-50 years, 28 patients aged 51-60 years, 41 patients aged 61-70 years, and 19 patients aged older than 70 years. Age-at-onset was analyzed based on clinical characteristics using the Kaplan-Meier method and were compared using the log-rank test. Sex, tumor-necrosis-metastasis (TNM) stage, and histology were not associated with age-atonset at statistically significant levels (Table 2).

\begin{tabular}{lcc}
\multicolumn{2}{c}{ Table 1. Onset age distribution in NSCLC patients. } & \\
\hline Age (years) & & No. of cases \\
\cline { 2 - 3 } & Male & Female \\
\hline$\leq 40$ & 4 & 3 \\
$41-50$ & 6 & 5 \\
$51-60$ & 16 & 12 \\
$60-70$ & 29 & 12 \\
$>70$ & 12 & 7 \\
\hline
\end{tabular}

The relationship between mtDNA genotype and age-at-onset was compared, and NSCLC patients were divided into 2 groups based on their genotype at each SNP site. The relationships between genotype and age-at-onset were analyzed using the log-rank test for all NSCLC patients at these sites. The SNP sites of nucleotides 200G/A and $16362 \mathrm{~T} / \mathrm{C}$ were examined for their association with age-at-onset using the log-rank test and were found to be significantly different $(\mathrm{P}<0.05)$. The nucleotide $207 \mathrm{G} / \mathrm{A}$ was identified for its association with age-at-onset and was found to be nearly significant $(\mathrm{P}=$ 0.060 ). The minor alleles $200 \mathrm{G}, 16362 \mathrm{C}$, and $207 \mathrm{~A}$ were associated with early onset of NSCLC (Table 3). 
Table 2. Clinical characteristics and their association with onset age in NSCLC patients.

\begin{tabular}{llccc}
\hline Characteristics & & No. of cases & Mean onset age (years) & P value \\
\hline Gender & & & & 0.443 \\
& Male & 67 & $61.21 \pm 11.21$ & 0.670 \\
\multirow{5}{*}{ TNM classification } & Female & 39 & $59.64 \pm 11.26$ & \\
& III & 27 & $60.93 \pm 11.21$ & 0.648 \\
Histology & IV & 79 & $60.53 \pm 11.26$ & 0.963 \\
& SQ $^{\mathrm{a}}$ & 35 & $62.94 \pm 8.47$ & \\
Chemotherapy & $\mathrm{AC}^{\mathrm{b}}$ & 71 & $59.49 \pm 12.22$ & \\
& No & 16 & $69.63 \pm 11.97$ & \\
& Yes & 90 & & \\
\hline
\end{tabular}

${ }^{\mathrm{a} S Q}$ : squamous cell carcinoma; ${ }^{\mathrm{b}} \mathrm{AC}$ : adenocarcinoma.

Table 3. Polymorphic sites and their association with onset age in NSCLC patients.

\begin{tabular}{lcccc}
\hline Polymorphic sites & & No. of cases & Age & P value \\
\hline 200 & G & 7 & $57.86 \pm 4.98$ & 0.043 \\
& A & 99 & $60.83 \pm 11.50$ & \\
& & & & 0.047 \\
20362 & C & 47 & $68.77 \pm 10.92$ & \\
& T & 59 & $52.12 \pm 11.28$ & 0.060 \\
& A & 8 & $60.75 \pm 8.07$ & \\
& G & 98 & & \\
\hline
\end{tabular}

\section{DISCUSSION}

Selected SNPs in the D-loop region have been examined for their ability to predict cancer risk and outcome in other types of tumor (Navaglia et al., 2006; Bai et al., 2007, 2008; Zhang et al., 2010b; Wang et al., 2007, 2008, 2011). In this study, we extended the analyses of previous studies to determine the relationships between age-at-onset and germline SNPs in a continuous sequence of mtDNA between nucleotides 583 and 16,190 in NSCLC patients. SNPs $200 \mathrm{~A} / \mathrm{G}, 207 \mathrm{G} / \mathrm{A}$, and 16362/C were identified for their association with age-at-onset and were statistically significant based on the log-rank test.

We examined D-loop SNPs in lung cancers (Ding et al., 2012a,b). In addition to acting as a predictor for cancer risk and outcome, SNPs in the D-loop can also predict age-at-onset in NSCLC patients. All 3 age-at-onset associated SNP sites were located in the hypervariable segment (HV) region of the D-loop, including nucleotides 200 and 207 located in HV-II and nucleotide 16,362 located in HV-I region. We identified outcome-associated SNPs in these regions for other cancers (Zhang et al., 2010a; Wang et al., 2011). The functional significance of hypervariable segments, which are mutational hotspots at which germline and tumor mtDNA mutations preferentially occur (Stoneking, 2000), is unknown; however, our data imply a role for these segments in cancer development.

The D-loop region of mtDNA is important for the regulation of mitochondrial genome replication and expression. SNPs in this region may affect mtDNA replication and lead to electron transport chain alteration, which is responsible for the release of high ROS produc- 
tion, nuclear genome damage, and cancer initiation and promotion (Bandy and Davison, 1990; Gille and Joenje, 1992). These SNPs may alter mitochondrial genome transcription, enhancing ROS production (Dement et al., 2007). This ROS-mediated mechanism may be associated with the age-at-onset of NSCLC.

Analysis of genetic polymorphisms in the D-loop region may help to identify patient subgroups that are at a higher risk of developing lung cancer, thereby helping to refine therapeutic decisions for these patients.

\section{ACKNOWLEDGMENTS}

Research supported by the National Natural Science Foundation of China \#30801384.

\section{REFERENCES}

Bai RK, Leal SM, Covarrubias D, Liu A, et al. (2007). Mitochondrial genetic background modifies breast cancer risk. Cancer Res. 67: 4687-4694.

Bandy B and Davison AJ (1990). Mitochondrial mutations may increase oxidative stress: implications for carcinogenesis and aging? Free Radic. Biol. Med. 8: 523-539.

Beal MF (1996). Mitochondria, free radicals, and neurodegeneration. Curr. Opin. Neurobiol. 6: 661-666.

Cao C, Zhang YM, Wang R, Sun SF, et al. (2011). Excision repair cross complementation group 1 polymorphisms and lung cancer risk: a meta-analysis. Chin. Med. J. 124: 2203-2208.

Dement GA, Maloney SC and Reeves R (2007). Nuclear HMGA1 nonhistone chromatin proteins directly influence mitochondrial transcription, maintenance, and function. Exp. Cell Res. 313: 77-87.

DiMauro S and Schon EA (2001). Mitochondrial DNA mutations in human disease. Am. J. Med. Genet. 106: 18-26.

Ding C, Li R, Wang P, Fan H, et al. (2012a). Sequence polymorphisms of the mitochondrial displacement loop and outcome of non-small cell lung cancer. Exp. Ther. Med. 3: 861-864.

Ding C, Li R, Wang P, Jin P, et al. (2012b). Identification of sequence polymorphisms in the D-Loop region of mitochondrial DNA as a risk factor for lung cancer. Mitochondrial DNA 23: 251-254.

Freedman ND, Leitzmann MF, Hollenbeck AR, Schatzkin A, et al. (2008). Cigarette smoking and subsequent risk of lung cancer in men and women: analysis of a prospective cohort study. Lancet Oncol. 9: 649-656.

Gille JJ and Joenje H (1992). Cell culture models for oxidative stress: Superoxide and hydrogen peroxidative versus normobaric heperoxia. Mutat. Res. 275: 405-414.

Lin G, Fang F, Yu XJ and Yu L (2011). Meta-analysis of the relationship between p21 Ser31 Arg polymorphism and lung cancer susceptibility. Genet. Mol. Res. 13: 2449-2456.

Nashikawa M, Nishiguchi S, Shiomi S, Tamori A, et al. (2001). Somatic mutation of mitochondrial DNA in cancerous and noncancerous liver tissue in individuals with hepatocellular carcinoma. Cancer Res. 61: 1843-1845.

Navaglia F, Basso D, Fogar P, Sperti C, et al. (2006). Mitochondrial DNA D-loop in pancreatic cancer: somatic mutations are epiphenomena while the germline 16519T variant worsen metabolism and outcome. Am. J. Clin. Pathol. 126: 593-601.

Parkin DM, Bray FI and Devesa SS (2001). Cancer burden in the year 2000. The global picture. Eur. J. Cancer 37 (Suppl 8): S4-S66.

Sanchez-Cespedes M, Parrella P, Nomoto S, Cohen D, et al. (2001). Identification of a mononucleotide repeats as a major target for mitochondrial DNA alterations in human tumors. Cancer Res. 61: 7015-7019.

Shadel GS and Clayton DA (1997). Mitochondrial DNA maintenance in vertebrates. Annu. Rev. Biochem. 66: 409-435.

Stoneking M (2000). Hypervariable sites in the mtDNA control region are mutational hotspots. Am J. Hum Genet. 67: 1029-1032.

Taanman JW (1999). The mitochondrial genome: structure, transcription, translation and replication. Biochim. Biophys. Acta 1410: 103-123.

Toyooka S, Mitsudomi T, Soh J, Aokage K, et al. (2011). Molecular oncology of lung cancer. Gen. Thorac. Cardiovasc. Surg. 59: 527-537.

Wang C, Zhang F, Fan H, Peng L, et al. (2011). Single nucleotide polymorphisms in the mitochondrial displacement loop and hepatocellular carcinoma outcome. Biochem. Biophys. Res. Commun. 406: 493-496. 
Wang L, Bamlet WR, de Andrade M, Boardman LA, et al. (2007). Mitochondrial genetic polymorphism and pancreatic cancer risk. Cancer Epidemiol. Biomarkers Prev. 16: 1455-1459.

Wang L, McDonnell SK, Hebbring SJ, Cunningham JM, et al. (2008). Polymorphism in mitochondrial genes and prostate cancer risk. Cancer Epidemiol. Biomarkers Prev. 17: 3558-3566.

Yang L, Yang G, Zhou M, Smith M, et al. (2009). Body mass index and mortality from lung cancer in smokers and nonsmokers: a nationally representative prospective study of 220,000 men in China. Int. J. Cancer 125: 2136-2143.

Yoneyama H, Hara T, Kato Y, Yamori T, et al. (2005). Nucleotide sequence variation is frequently in the mitochondrial DNA displacement loop region of individual human tumor cell. Mol. Cancer Res. 3: 14-20.

Zhang R, Wang R, Zhang F, Zhang R, et al. (2010a). Single nucleotide polymorphisms in the mitochondrial displacement loop and outcome of esophageal squamous cell carcinoma. J. Exp. Clin. Cancer Res. 29: 155.

Zhang R, Zhang F, Wang C, Wang S, et al. (2010b). Identification of sequence polymorphism in the D-loop region of mitochondria DNA as a risk factor for hepatocellular carcinoma with distinct etiology. J. Exp. Clin. Cancer Res. 29: 130. 